ORIGINAL RESEARCH

\title{
Assessment of Cervical Volume at 19-22 Weeks for Predicting a Prolonged Pregnancy
}

Nabil Manzour ${ }^{1}$, Enrique Chacon ${ }^{2}$, Itsaso Areta ${ }^{3}$, Isabel Carriles ${ }^{4}$, Isabel Brotons ${ }^{5}$, Juan L Alcázar ${ }^{6}$

\begin{abstract}
Objective: To determine whether the transvaginal ultrasonographic measurement of the cervical volume at 19-22 weeks could predict a postterm pregnancy.

Materials and methods: This work involves a retrospective case-control study comprising 44 women who delivered beyond 41 weeks and 87 women who delivered at term (37-40+6 weeks), matched by age and parity. All of them had undergone cervical length measurement and cervical volume estimation at 19-22 weeks.

Results: Patients' median of age was 35 years in term gestations and 34.5 years in prolonged pregnancies $(p=0.313)$. The mean of gestational age during delivery in the term gestation group was 275.41 days vs 289.34 days on prolonged gestations $(p<0.001)$. We did not observe differences in the mean cervical volume between term delivery $\left(37.37 \mathrm{~cm}^{3}, 95 \% \mathrm{Cl}: 34.59-40.14\right)$ and those who had post-term delivery (38.06 $\left.\mathrm{cm}^{3}, 95 \% \mathrm{Cl}: 33.34-42.77\right)(p=0.788)$. In addition, we did not find differences in the median cervical length (39.0 mm vs $\left.37.0 \mathrm{~mm}\right)(p=0.610)$. Conclusion: It seems that there is no relationship between the cervical volume measured in the ultrasound of 20-week gestation and the prolongation of pregnancy beyond week 41.
\end{abstract}

Keywords: Cervical length, Cervical volume, Post-term, Pregnancy, Volume.

Donald School Journal of Ultrasound in Obstetrics and Gynecology (2019): 10.5005/jp-journals-10009-1592

\section{INTRODUCTION}

Prolonged pregnancy occurs in approximately $10 \%$ of all singleton pregnancies and it is associated with an increased risk of fetal macrosomia, intrapartum fetal heart rate abnormalities, meconium staining, perinatal death, and cesarean delivery. ${ }^{1-3}$ An early prediction of this condition is important because several methods to decrease the rate of prolonged pregnancy, such as membrane stripping and outpatient prostaglandin therapy, have already been proposed. ${ }^{4,5}$ Therefore, there has been considerable interest in the development of tests for the prediction of a prolonged pregnancy. These tests include fetal fibronectin, cytokine, or nitric oxide concentrations in cervicovaginal secretions, as well as cervical length (as determined by ultrasonography). ${ }^{6-10}$

Cervical length measured by the transvaginal ultrasound in the second trimester has been known to be effective in identifying pregnancies at a high risk for a spontaneous preterm delivery. ${ }^{11-16}$

Recent studies have reported on the importance of ultrasonographic examinations of the uterine cervical length in predicting post-term pregnancies. ${ }^{17}$ However, to the best of our knowledge, there are no ultrasound studies in the current literature analyzing the relationship between the cervical volume and prolonged pregnancies.

The aim of this study was to determine whether the transvaginal ultrasonographic measurement of the cervical volume at 19-22 weeks could predict post-term pregnancies.

\section{Materials and Methods}

After obtaining the approval of the ethics committee of the Clinica Universidad de Navarra (CUN), a retrospective case-control study was carried out. The women participating in the study have been evaluated at the Gynecology and Obstetrics Department of this tertiary care university hospital at least from 19th to 22th weeks until

\footnotetext{
${ }^{1-6}$ Department of Obstetrics and Gynecology, Clinica Universidad de Navarra, School of Medicine, Pamplona, Spain

Corresponding Author: Juan L Alcázar, Department of Obstetrics and Gynecology, Clínica Universidad de Navarra, School of Medicine, Pamplona, Spain. Phone: +34-948-296234, e-mail: jlalcazar@unav.es

How to cite this article: Manzour N, Chacon E, et al. Assessment of Cervical Volume at 19-22 Weeks for Predicting a Prolonged Pregnancy. Donald School J Ultrasound Obstet Gynecol 2019;13(3):99-102.

Source of support: Nil

Conflict of interest: None
}

delivery. These women had signed an informed consent accepting that their clinical data could be used for this research.

At our institution, all pregnant women are offered a routine ultrasound examination at 19th-22th weeks for a fetal anomaly scan and for performing cervical biometry and volumetry. At CUN, a pregnancy is considered to be prolonged after week 41 , when an induction of labor is advised.

Inclusion criteria for this study were as follows: live fetus, intact amniotic membranes, no fetal pathology, gestational age confirmed by ultrasound at 12th week, no previous uterine interventions, pregnancy had been followed at least from 19th to 22th weeks of gestation to delivery at our institution, and a screening ultrasound had been done between 19th week and 22th week, including cervical biometry.

Exclusion criteria were as follows: an induction of labor or a cesarean section for any reason before week 41 and no measurement of cervical volume in 19th-22th week scan.

Patients belonging to the cases group were a consecutive series of women who delivered at 41-42 weeks at our institution between April 2014 and August 2017. The control-group patients were randomly selected using the immediate woman who delivered 
spontaneously at 37-40 weeks prior to the cases-group patients, matched by age ( \pm 1 year old) and parity (same parity).

The measurement of the cervical length and volume was performed using the transvaginal ultrasound between week 19 and week 22 by different sonographers from the Department of Obstetrics and Gynecology, who are properly trained for this type of ultrasound examinations. The ultrasound machine used for all measurements was a Voluson E8 (General Electric Healthcare, Milwaukee, WI, USA) equipped with a 5-9 MHz endovaginal probe. The women were examined at the lithotomy position, with an empty bladder according to the methodology described elsewhere. ${ }^{18,19}$ Briefly, the ultrasound probe is inserted slowly into the vagina, avoiding excessive pressure, which can artificially lengthen the cervix. After obtaining a satisfactory image of the cervix, the probe was removed until the image became blurred. Then, the probe moved forward gradually with just enough pressure to restore a satisfactory image. A sagittal view of the cervix was obtained where the internal os, the cervical canal, and the external os were all visible simultaneously. The image was enlarged so that the cervix occupies approximately $75 \%$ of the screen. The cervical length was measured from the external to the internal cervical os, being careful to include only the segment of the cervix canal that was bordered by the endocervical mucosa. Three measurements were performed and the average of them was used for our analysis (Fig. 1). Once 2D

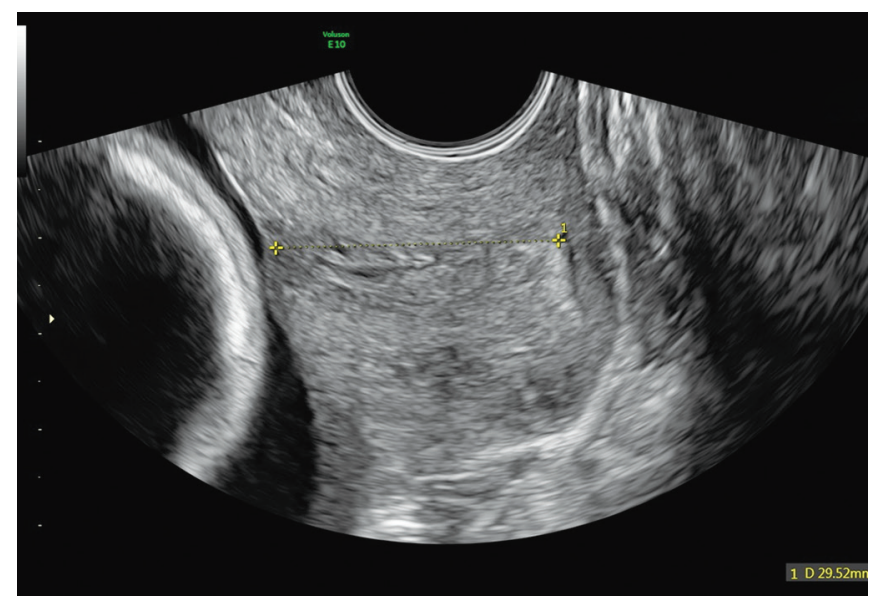

sonography was done, a 3D mode was activated and the cervix was centralized within the 3D sector appearing on ultrasound screen. Then, a 3D volume was acquired by using the maximum angle $\left(120^{\circ}\right)$. The probe was rotated $90^{\circ}$, and a second $3 \mathrm{D}$ volume was acquired in the transverse plane. Both 3D volumes were stored for a subsequent analysis. As stated before, all 2D measurements and 3D volume acquisitions were performed by several trained sonographers.

The 4D View program (General Electric Healthcare, Zipf, Austria) was used to measure the cervical volume. The program was set to manual. The drawing started from a longitudinal section through the cervix. The rotation steps were set at $30^{\circ}$ and six contours of the cervix were drawn manually using the cursor of the system. Once all contours had been drawn, the volume of the cervix was calculated automatically and expressed in cubic centimeters ${ }^{19,20}$ (Fig. 2).

All cervical volume measurements were performed by the same examiner. This examiner was blinded to whether the patients belonged to the group of cases or that of controls.

The following variables were studied: patient's age, number of past pregnancies, number of past abortions, late abortions in previous pregnancies, a threatened abortion in the first trimester of the current gestation, number of deliveries, date of the ultrasound at 19th-22th week (in days), cervical length measured at 19th-22th week ultrasound scan (in $\mathrm{mm}$ ), cervical volume measured at 19th-22th week ultrasound scan (in $\mathrm{cm}^{3}$ ), hypothyroidism in the pregnancy studied, diabetes in the gestation studied, gestational age at the time of delivery, the gestation studied in days, type of delivery, and newborn's weight.

\section{Statistical Analysis}

The Kolmogorov-Smirnov test was used to determine the distribution of data in continuous variables. The categorical variables are presented as absolute number and percentage, and compared with the Pearson's $X^{2}$ test. The continuous variables were compared using the one-way variance analysis (ANOVA) or the Mann-Whitney $U$ test, depending on the distribution of data.

The statistical power analysis was not performed and $p<0.05$ is considered statistically significant. Statistical analyses were conducted using SPSS 15.0 (SPSS Inc., Chicago, IL).

Fig. 1: Cervical length measurement by the transvaginal ultrasound
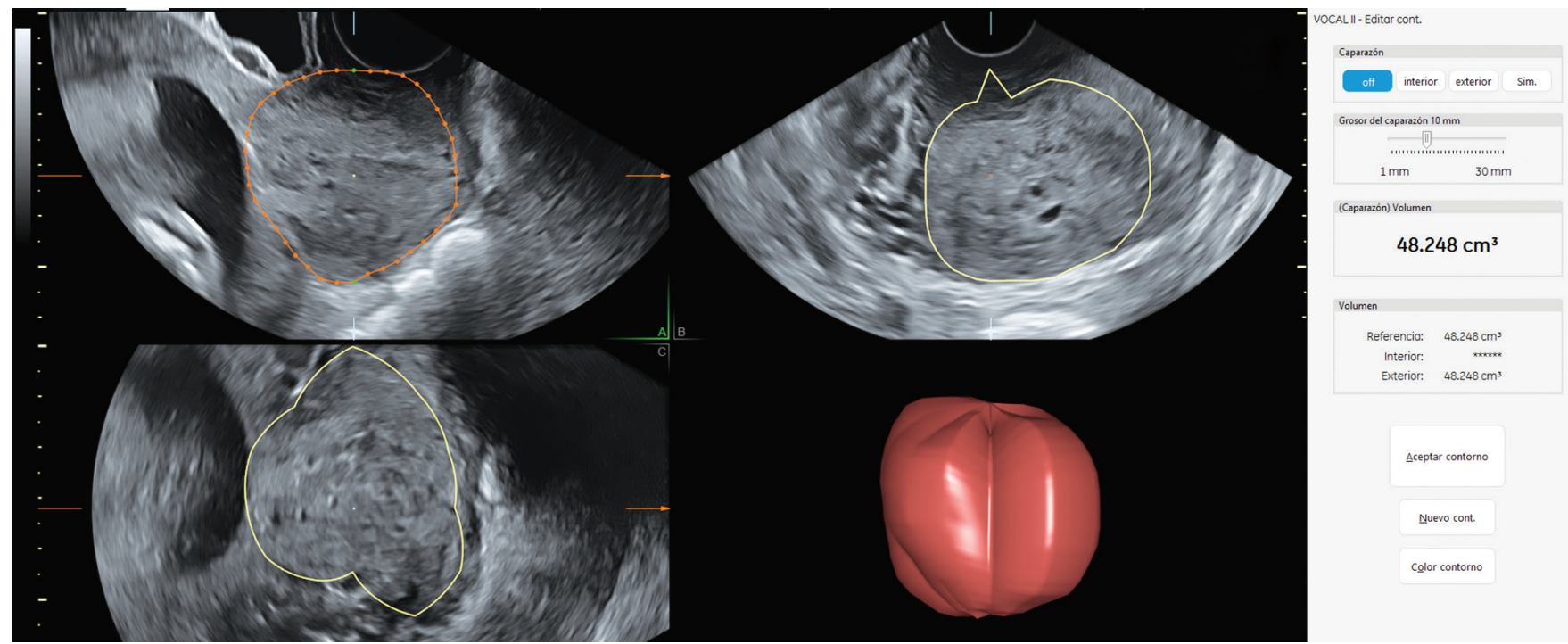

Fig. 2: Cervical volume estimation using the 3D ultrasound 


\section{Results}

An estimated 105 term gestation patients (37-40+6 weeks) and 104 post-term patients (41 weeks and over) were enrolled for the study. Although there is an indication to perform the measurement of the cervical volume in all ultrasound examinations at 19 - to 22 weeks scan, this was not carried out in all patients. Thus, we had cervical volume data from 131 patients: 44 cases and 87 controls.

Most continuous variables did not follow a normal distribution, except the weight of the newborn, date of the ultrasound of the week 19-22, cervical volume, and gestational age at the time of delivery.

When comparing the demographic characteristics between both groups, we found only statistical differences for the gestational age at the time of delivery, the weight of the newborn, and the rate of vaginal delivery (Table 1).

When we compared cervical volume and length measured by ultrasound at week 19-22 of gestation, we did not observe significant differences between both groups (Table 2).

\section{Discussion}

In the present study, we have observed that both median cervical length and volume measured at 19- to 22-weeks scan using the transvaginal ultrasound are similar for prolonged gestations and term gestations.

Previous studies have used the sonographic evaluation of the cervical length to predict the onset of a preterm labor. ${ }^{13-16}$ However, there are scanty and controversial data about the relationship of this measurement and a post-term pregnancy. ${ }^{17,21-24}$
Firstly, this topic deserves attention because the measurement of cervical volume in the second trimester can identify patients at a high risk for a prolonged pregnancy; the incidence of a prolonged pregnancy or the risks associated with a prolonged pregnancy will be reduced because a simple method to promote the spontaneous onset of a labor (i.e., membranes stripping and outpatient prostaglandin therapy) was already proposed. Secondly, these data might be used in individualizing the timing of an elective cesarean section rather than in the performance of this operation at 37 weeks-for example, delaying a cesarean section until 39 weeks if there were any other indications. Thirdly, from the patients' point of view, these data may give patients some information to arrange their social activities and to deal with their anxieties.

Suh et al. found that the cervical length measured at 20-24 weeks was not a predictor of prolonged pregnancies. ${ }^{17}$ Our results are in agreement with these data.

However, Donelan et al. reported in a larger series that women with a cervical length above the third quartile, measured at 18-24 weeks had a higher risk for a prolonged pregnancy. ${ }^{21}$

Boeling et al. found that women with a cervical length $>37 \mathrm{~mm}$ (beyond first quartile) during the second trimester had a twofold increase of a prolonged pregnancy than women with a cervical length $<37 \mathrm{~mm} .{ }^{22}$ However, Van der Ven et al. reported that a postterm pregnancy was more likely (OR: 2.02) when the cervical length was $>45 \mathrm{~mm}$ between $16+0$ and $21+6$ weeks of gestation. ${ }^{23}$ More recently, Thangaraj et al. reported similar findings: $90 \%$ of women in their series with a cervical length $>4 \mathrm{~cm}$ in midtrimester $(n=50)$ delivered beyond the 40th week of gestation. ${ }^{24}$

Table 1: Demographic characteristics in term and post-term pregnancies

\begin{tabular}{llll}
\hline & Term gestations & Prolonged gestations & p value \\
\hline Gestational age at the time of delivery days & $275.41(273.84-276.98)^{*}$ & $289.34(288.66-290.02)^{*}$ & 0.001 \\
Weight of the newborn in kilograms & $3.30(3.20-3.40)^{*}$ & $3.61(3.49-3.74)^{*}$ & 0.001 \\
Patients' age (years old) & $35(5)^{* *}$ & $34.5(7)^{* *}$ & 0.313 \\
Number of pregnancies & $2(2)^{* *}$ & $2(2)^{* *}$ & 0.520 \\
Number of abortions & $0(1)^{* *}$ & $0(0)^{* *}$ & $1(1)^{* *}$ \\
Number of deliveries & $2(2)^{* *}$ & $143(4)^{* *}$ & 0.540 \\
Date of the ultrasound of weeks 19-22 (days) & $142(4)^{* *}$ & $0(0)^{\dagger}$ & 0.510 \\
Late abortions in previous pregnancies & $3(3.4)^{\dagger}$ & $1(2.3)^{\dagger}$ & 0.480 \\
Threatened abortion in the first trimester & $4(4.6)^{\dagger}$ & $8(18.2)^{\dagger}$ & 0.550 \\
Hypothyroidism & $17(19.5)^{\dagger}$ & $2(4.5)^{\dagger}$ & 0.663 \\
Diabetes & $3(3.4)^{\dagger}$ & $34(77.3)^{\dagger}$ & 1.000 \\
Vaginal delivery & $79(90.8)^{\dagger}$ & $7(15.9)^{\dagger}$ & 1.000 \\
Premature rupture of membranes & $21(24.1)^{\dagger}$ & 0.034 \\
\hline
\end{tabular}

*Mean with 95\% confidence interval in parentheses

**Median with interquartile range in parentheses

${ }^{\dagger}$ Absolute number, percentage in parentheses

Table 2: Cervical length and volume in term and post-term gestations as measured at 19-22 weeks

\begin{tabular}{llll}
\hline & Term gestations & Prolonged gestations & p value \\
\hline $\begin{array}{l}\text { Cervical volume measured in the ultrasound of weeks } \\
\text { 19-22 in cm }\end{array}$ & $37.37(34.59-40.14)^{*}$ & $38.06(33.34-42.77)^{*}$ & 0.788 \\
$\begin{array}{l}\text { Cervical length measured in the ultrasound of weeks } \\
19-22 \text { in mm }\end{array}$ & $39(9)^{* *}$ & $37(7.75)^{* *}$ & 0.610 \\
\hline *Mean with 95\% confidence interval in parentheses & & \\
$*$ **Median with interquartile range in parentheses & &
\end{tabular}


Owing to the controversial results related to the cervical length and its relationship with prolonged pregnancies, we wondered whether the cervical volume estimation could be useful for this purpose. We have observed that the cervical volume, as measured in the midtrimester ultrasound scan, is not useful to predict prolonged pregnancies.

The main strength of our study is its originality. To the best of our knowledge, this study is the first to describe the possible changes in the cervical volume at the mid-gestation and their relationship with a prolonged delivery.

However, we acknowledge that our study has several limitations. On the one hand, the retrospective design implies that there are variables that were not measured in all women, mainly cervical volume. Consequently, 78 patients had to be excluded. In addition, it is well known that parous women had slightly larger cervical volumes throughout the pregnancy than did nulliparous women. ${ }^{19}$ In our study, we included parous women and this could have potentially affected the results because primiparity and prior prolonged pregnancy are the most common identifiable risk factors for the prolongation of pregnancies.

In conclusion, with the results obtained, it seems that there is no relationship between the cervical volume measured in the midtrimester ultrasound and prolonged pregnancies. However, further studies are needed to confirm or refute these findings.

\section{References}

1. Rand L, Robinson JN, et al. Post-term induction of labor revisited. Obstet Gynecol 2000;96:779-783. DOI: 10.1016/S0029-7844(00)01002-4.

2. Sanchez-Ramos $L$, Olivier F, et al. Labor induction versus expectant management for postterm pregnancies: a systematic review with meta-analysis. Obstet Gynecol 2003;101:1312-1318. DOI: 10.1097/00006250-200306000-00029.

3. ACOG Practice Bulletin. Clinical management guidelines for obstetricians-gynecologists. Number 55, September 2004 (replaces practice pattern number 6, October 1997). Management of Postterm Pregnancy. Obstet Gynecol 2004;104:639-646. DOI: 10.1097/00006250-200409000-00052.

4. Magann EF, McNamara MF, et al. Can we decrease postdatism in women with an un-favorable cervix and a negative fetal fibronectin test result at term by serial membrane sweeping? Am J Obstet Gynecol 1998;179:890-894. DOI: 10.1016/S0002-9378(98)70184-X.

5. McKenna DS, Costa SW, et al. Prostaglandin E2 cervical ripening without subsequent induction of labor. Obstet Gynecol 1999;94: 11-14. DOI: 10.1016/S0029-7844(99)00244-6.

6. Imai $M$, Tani A, et al. Significance of fetal fibronectin and cytokine measurement in the cervico-vaginal secretions of women at term in predicting term labor and post-term pregnancy. Eur J Obstet Gynecol Reprod Biol 2001;97:53-58. DOI: 10.1016/S0301-2115(00)00483-8.

7. Lockwood CJ, Moscarelli RD, et al. Low concentrations of vaginal fetal fibronectin as a predictor of deliveries occurring after 41 weeks. Am J Obstet Gynecol 1994;171:1-4. DOI: 10.1016/S0002-9378(94)70068-0.

8. Vaisanen-Tommiska $M$, Nuutila $M$, et al. Cervical nitric oxide release in women postterm. Obstet Gynecol 2004;103:657-662. DOI: 10.1097/01. AOG.0000115509.10605.64.

9. Ramanathan G, Yu C, et al. Ultrasound examination at 37 weeks' gestation in the prediction of pregnancy outcome: the value of cervical assessment. Ultrasound Obstet Gynecol 2003;22:598-603. DOI: 10.1002/uog.913.

10. Vimercati A, Greco P, et al. The value of ultrasonographic examination of the uterine cervix in predicting post-term pregnancy. J Perinat Med 2001;29:317-321. DOI: 10.1515/JPM.2001.045.

11. lams JD, Goldenberg RL, et al. The length of the cervix and the risk of spontaneous premature delivery. National Institute of Child Health and Human Development Maternal Fetal Medicine Unit Network. N Engl J Med 1996 Feb 29;334(9):567-572. DOI: 10.1056/ NEJM199602293340904.

12. Carvalho MH, Bittar RE, et al. Cervical length at 11-14 weeks' and 22-24 weeks' gestation evaluated by transvaginal sonography, and gestational age at delivery. Ultrasound Obstet Gynecol 2003;21: 135-139. DOI: 10.1002/uog.32.

13. Hassan SS, Romero R, et al. Patients with an ultrasonographic cervical length $<$ or $=15 \mathrm{~mm}$ have nearly a $50 \%$ risk of early spontaneous preterm delivery. Am J Obstet Gynecol 2000;182:1458-1467. DOI: 10.1067/mob.2000.106851.

14. Murakawa $\mathrm{H}$, Utumi T, et al. Evaluation of threatened preterm delivery by transvaginal ultrasonographic measurement of cervical length. Obstet Gynecol 1993;82:829-832.

15. Heath VC, Southall TR, et al. Cervical length at 23 weeks of gestation: prediction of spontaneous pre-term delivery. Ultrasound Obstet Gynecol 1998;12:312-317. DOI: 10.1046/j.1469-0705.1998.12050312.x.

16. To MS, Skentou C, et al. Cervical length and funneling at 23 weeks of gestation in the prediction of spontaneous early preterm delivery. Ultrasound Obstet Gynecol 2001;18:200-203. DOI: 10.1046/j.14690705.2001.00437.x.

17. Young HS, Kyo HP, et al. Prediction of prolonged pregnancy in nulliparous women by transvaginal ultrasonographic measurement of cervical length at 20-24 weeks and 37 weeks. J Korean Med Sci 2007;22:89-93. DOI: 10.3346/jkms.2007.22.1.89.

18. Rovas $L$, Sladkevicius $P$, et al. Reference data representative of normal findings at two-dimensional and three-dimensional gray-scale ultrasound examination of the cervix from 17 to 41 weeks' gestation. Ultrasound Obstet Gynecol 2006;27:392-402. DOI: 10.1002/uog. 2658.

19. Rovas $L$, Sladkevicius $P$, et al. Reference data representative of normal findings at three-dimensional power Doppler ultrasound examination of the cervix from 17 to 41 gestational weeks. Ultrasound in Obstetrics and Gynecology 2006;28:761-767. DOI: 10.1002/ uog.2857.

20. Arribas S, Alcázar JL, et al. Three-Dimensional Transvaginal Sonography and Magnetic Resonance Imaging for Local Staging of Cervical Cancer. Journal of Ultrasound in Medicine 2016;35:867-873. DOI: 10.7863/ultra.15.05071.

21. Donelan EA, Grobman WA, et al. Association of Second-Trimester Cervical Length with Prolonged Pregnancy. Obstet Gynecol 2015;126:534-538. DOI: 10.1097/AOG.0000000000000976.

22. Boelig RC, Orzechowski KM, et al. Second trimester cervical length and prolonged pregnancy. J Matern Fetal Neonatal Med 2016;29:4088-4091. DOI: 10.3109/14767058.2016.1159191.

23. van der Ven AJ, van Os MA, et al. Midpregnancy Cervical Length in Nulliparous Women and its Association with Postterm Delivery and Intrapartum Cesarean Delivery. Am J Perinatol 2016;33:40-46. DOI: 10.1055/s-0035-1556067.

24. Thangaraj JS, Habeebullah S, et al. Mid-Pregnancy Ultrasonographic Cervical Length Measurement (A Predictor of Mode and Timing of Delivery): An Observational Study. J Family Reprod Health 2018;12: 23-26. 